Article

\title{
Exploring Community Symbiotic Tourism Programs for the Utilization and Conservation of Ecology in Lava Stony Forest (Gotjawal) of Jeju Island, Korea
}

\author{
Chang-Yu Hong ${ }^{1, * \mathbb{D}}$, Ran Yoon ${ }^{2} \mathbb{D}$, Jung-Don Hwang ${ }^{3}$ and Min-Seok Jwa ${ }^{4, *}$ \\ 1 Department of Urban Environmental Research, Jeju Research Institute, Jeju 63147, Korea \\ 2 School of Environmental Planning, Seoul National University, Seoul 08826, Korea; ranyoon525@gmail.com \\ 3 Department of Economics, Jeju National University, Jeju 63243, Korea; lifeisreal@empas.com \\ 4 Department of Future Strategy, Jeju Research Institute, Jeju 63147, Korea \\ * Correspondence: 503changyu@gmail.com (C.-Y.H.); jms69@jri.re.kr (M.-S.J.); Tel.: +82-64-729-0522 (M.-S.J.)
}

Received: 22 September 2020; Accepted: 9 October 2020; Published: 12 October 2020

\begin{abstract}
According to our research findings, the future-oriented conservation of natural ecological resources in the Sahn-Yang Gotjawal area should be shaped and formed in a sustainable ecological way to improve the economy of the Sahn-Yang area. This study covers a systematic and quantitative assessment of the function, design, and purpose of establishing a regional eco-tourism program using the Sahn-Yang Gotjawal (Volcanic Lava Stony Forest) natural resources of Jeju Island, South Korea. Although citizens' interest in Jeju Gotjawal has increased, reckless exploration and damage still occurred. There were improvements on the movement to preserve geological features and the ecosystem. However, unfortunately the negative effects of only seeking development opportunities without regard to the environment was realized as well. Through strengths, opportunities, aspirations, results (SOAR) analysis, various potential situations and conditions using the Sahn-Yang Gotjawal resources were analyzed with quantitative and qualitative techniques. In addition, through media analysis, the benefits and opportunities that the Gotjawal resources could provide the citizens were identified and the results were combined with SOAR analysis to present an integrated vision. In order to make wise use of Sahn-Yang Gotjawal, such as conserving, sustaining, and creating accessibility (guided by SESs), it is necessary to concentrate on the 'strengths' such as creating and maximizing attractions which can develop management programs such as preservation research and monitoring, as well as establishing sustainable usage of facilities such as visitors' gathering spaces, exploration activities, and convenient facilities.
\end{abstract}

Keywords: Jeju Gotjawal; eco-tourism; community development; ecological resource management; Jeju Island; socio-ecological systems

\section{Introduction}

Eco-tourism has been promoted and widely adopted as a strategy for funding conservation initiatives, while at the same time contributing to the socioeconomic development of host communities and maximizing quality tourism experiences [1]. A more strict definition of eco-tourism requires that travel be environmentally and socially responsible, funds and support for conservation efforts are generated, and benefits to local host communities are provided [2-5]. Parks and protected areas are common eco-tourism destinations [6]. According to Stronza et al. [7], eco-tourism is a travel pattern for a well-preserved nature conservation zone. This means that community residents gain economic benefits through conservation without changing the ecosystem, and tourists increase their understanding of culture, environment, and natural history [7]. Many researchers pay attention to 
eco-tourism based on an interactive link between humans and nature [8]. In order to have better eco-tourism, stakeholders and related actors for the eco-tourism projects should accept and understand both the people and nature surrounding the tourism [9].

Some previous researchers have noted that conventional approaches to tourism resources and ecosystem management do not always work and may even make those issues worse [10]. The combination of powerful centralized institutions and functionally specialized divisions of resource exploitation is part of the ideology of conventional tourism development practices [11]. However, not only does this developmentalism ideology hinder tourism resource management reform, but the accompanying political interests and economic ideologies in tourism make adaptive social change difficult as well [12]. Thus, scholars began to seek integrated models reflecting more environmental and sustainable tourism resource management based upon interdisciplinary, adaptive, and complex systems [13]. As a result of these efforts, many scholars instigated the use of a new term, 'social-ecological systems (SESs).' Thus, we argue that SESs can provide solutions for the concerns of how the ecological system should be linked to cultural and social systems in managing regional tourism and conserving our natural resource [14].

Scholars such as Ostrom wanted to answer the question of how we can have a framework that includes both people and nature. The answer was social ecological systems [15]. Social-ecological systems (SESs) are complex adaptive-systems including social (human) and ecological (bio-physical) approaches, with interaction between both approaches in resiliency and sustainability [16]. In terms of SESs, scholars expound the integrated concept of human-in-nature [17]. In particular, SESs refer to the linked view as delineation between social and ecological systems in the natural environment [18]. Social systems deal with policies, property rights, culture, and local knowledge for environmental resources [19]. In addition, SESs include components such as resilience, sustainability, governance, robustness, and adaptive management [20].

In the perspective of SESs, ecological resilience is one of the main components for successful eco-tourism because it indicates predictability as well as giving clear feedback on the ecological degradation [21]. Ecological resilience can explain the magnitude or scale of disturbance that can be absorbed before the system's structure is compromised by the shift in external conditions resulting from human impact [22]. This is a fundamentally dissimilar view of science, in which determining causal effects and making predictions are not simple matters at all. In fact, systems are better understood as complex, non-linear, multi-equilibrium and self-organizing [23].

Concerning eco-tourism, SESs can depict the formation of governance linking various interests as well as different levels and scales [24]. The SESs are framed at various scales regarding politics and ecology [25]. The role and function of governance can determine successful SESs. According to Zia et al. [26], management of social-ecological systems contributes to establishing complex governance processes led by community members and cross-scale institutional arrangements that are mediated through politics of multiple scales [26]. SESs provide key insights to network policymaking and governance building for ecosystem conservation and community development through eco-tourism [27].

Through the lens of SES, we could reify the concept of eco-tourism. The purpose of eco-tourism is to understand nature by observing and experiencing the natural scenery and cultural resources. Therefore, eco-tourism activities will provide professional education programs and community development agendas to enhance the understanding of nature and culture in the target area, embrace wild animals and plants, and create experience programs to enhance harmony with nature through tourism [28]. The development and management techniques of eco-tourism are often used to minimize the adverse effects of current large-scale tourism development, such as diminished natural scenery and destruction of ecological resources [29]. In other words, in order to minimize the impact on the natural ecosystem, eco-tourism that pursues a sense of ecological sustainability and minimal consumption of natural resources will be required. 
Jeju, which seeks to become a global environmental hub, holds a long-term vision to provide the optimal arena for people and nature to coexist. Among the natural ecological resources that make up Jeju is the volcanic lava stony forest area of Jeju referred to as "Gotjawal". Jeju Gotjawal is a unique natural environment created by the volcanic activities, which formed Jeju Island, and refers to ecosystems, such as forests created on rocky lava. Many environmental policymakers and planners in Jeju are trying to develop programs such as Gotjawal eco-tourism to revitalize the local economy while preserving the Gotjawal resources.

Gotjawal, created by volcanic lava flows, is home to a variety of ferns, including Jeju Gosarisam (Jeju grape fern), an endangered species. Gotjawal is an important repository of ecosystems that represent Jeju [30]. As environmental damage occurred due to the modern development of Gotjawal, the Jeju government is pushing for various measures and research projects to preserve and manage the economically valuable Gotjawal area efficiently and systematically.

At the same time, it is necessary to discover regional harmonious eco-tourism projects between ecology and humans by methodically preserving the unique natural environment of Jeju Sahn-Yang Gotjawal and preparing a broad plan through strengthening the adaptive capacity of local residents. We define the adaptive capacity under the concept, socio-ecological systems (SESs). Therefore, this study presents a means for achieving the two goals of regional development and conservation of natural ecological resources by utilizing the resources of Gotjawal in Jeju's Sahn-Yang area by taking a frame of SESs.

\section{Study Area}

\subsection{The Location of Gotjawal and Sahn-Yang Gotjawal in Jeju Island}

Jeju Island's Gotjawal (Figure 1) are divided into four major groups: Jocheon-Hamdok Gotjawal, Gujwa-San Gotjawal, Hankyung-Andeok Gotjawal and Aewol Gotjawal. Hankung-Andeok Gotjawal is subdivided into Weolim-Sinpyeong Gotjawal and Sangchang-Hawsan Gotjawal. Sahn-Yang Gotjawal is located in Weolim-Sinpyeong Gotjawal. In particular, Sahn-Yang Gotjawal is located on the edge of the Gotjawal area where it extends to the Southeast of Hankung-myeon in Jeju. There are popular tourist destinations such as Jeoji Gotjawal (also called Cheongsu Gotjawal), and the recently established Jeju Gotjawal Provincial Park.

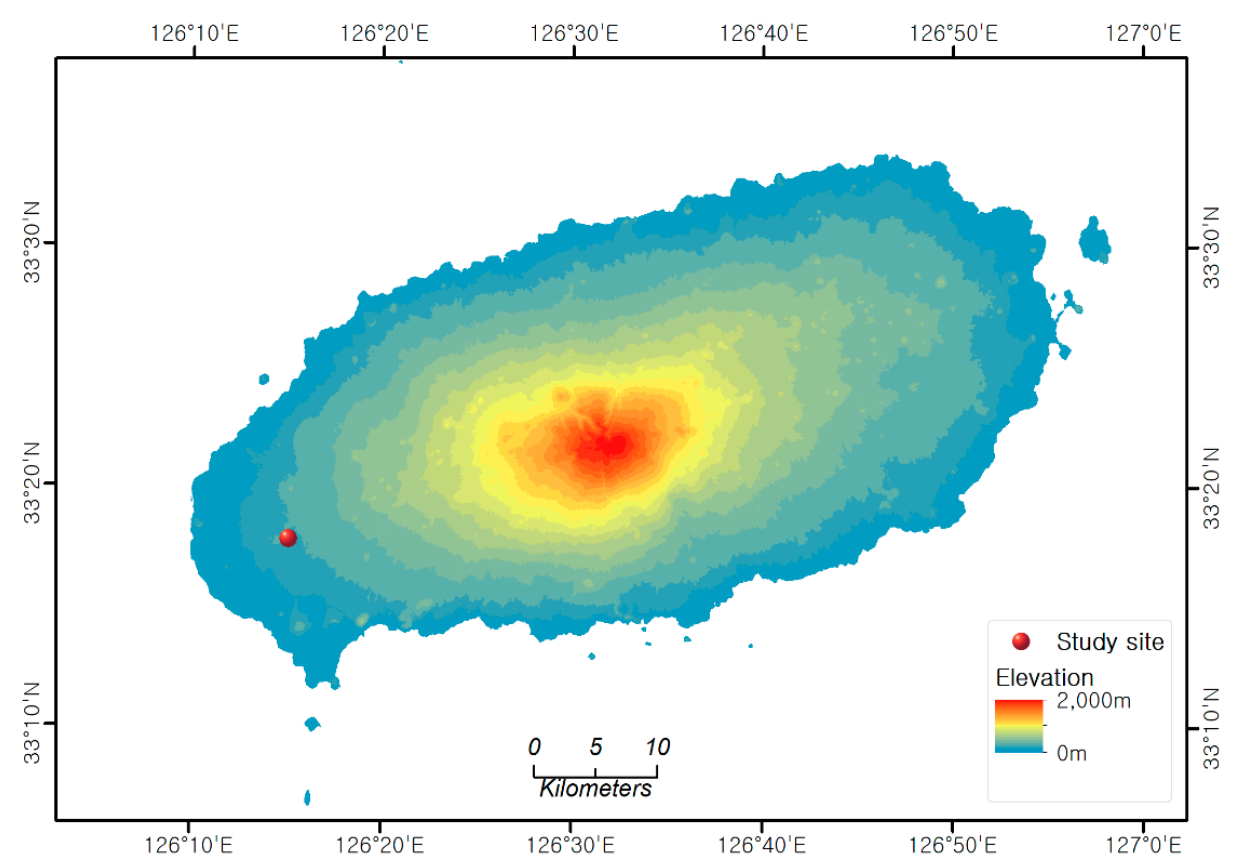

Figure 1. Topographic Map of Study Site Location of Sahn-Yang Gotjawal in Jeju Island. 


\subsection{The Wide Area Topography of Sahn-Yang Gotjawal}

In the southwestern area of Jeju Island where the Sahn-Yang Gotjawal is located, the entire area forms a gradual coastal and mountainous region with an increasing slope past the mountainous terrain. (Figure 1) Gotjawal is a lush greenery on Jeju Island and is a local term that is used for the area where trees and vines are entangled on a rocky hillside mostly under the mountains [31]. It is the only place in the world where tropical northern limit plants and southern Korea limit plants coexist. When volcanoes erupt, high-viscosity lava is divided into large and small rock masses and forms a rough terrain. On top of that, natural forest vines and mixed vegetation are present [32] (Figure 2). When it rains, the underground water accumulates, and it is considered the main source of spring water [33]. Gotjawal is a microclimate area that can sustain an even temperature and provides a stable habitat for wild animals and plants [30]. The amount of phytoncide released from plants in Gotjawal is high so it can prevent pathogen infection, purify air, remove odors, and even heal visitors.

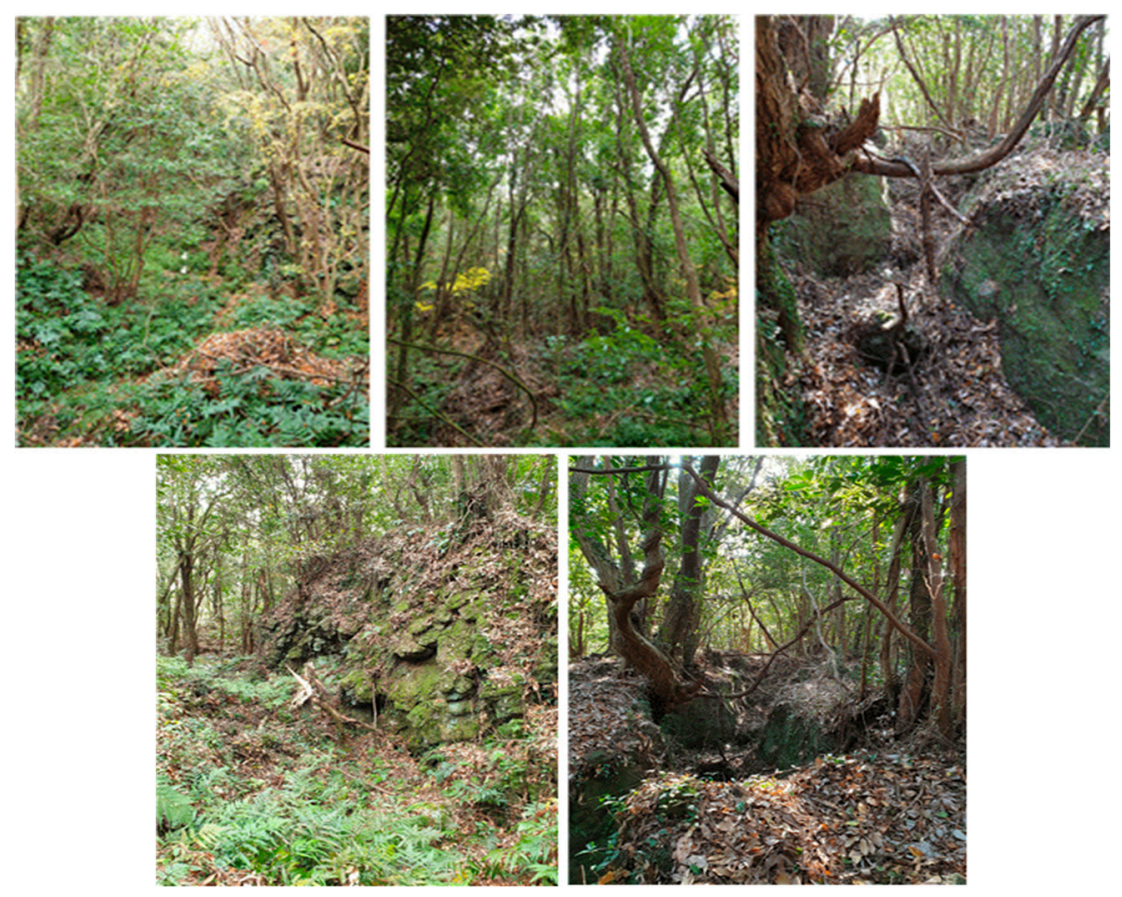

Figure 2. Topography in Sahn-Yang Gotjawal [32].

\subsection{Geospatial Geography Characteristics of Sahn-Yang Gotjawal as an Ecological Formation Site}

Past studies from geological points of view in the 1990s confirmed that the Gotjawal area was a lava region characterized by typical rock masses $A a$. The lava flows distributed in the Gotjawal area were named "Gotjawal Lava" [32]. Recently, it was reported that Gotjawal is developing not only in Aa, but also in the Pahoehoe area [33]. It has been reported that the surface characteristics of lava flows that make up one of the Gotjawal regions are not only showing various forms such as $A a$, Pahoehoe, and transitional lava stone forms, but also formulations with no special connection with the components of magma were observed [31,34]. Meanwhile, Sahn-Yang Gotjawal, located the bottom of the Wolin-Sinpyong Gotjawal area [32] consists of Pahoehoe type lava flow and developed in the form of a cave depression, lava embankment, or tumulus. These depressions show particular differences in temperature and humidity from the surrounding area and can also be the reason for Gotjawal's unique ecosystem formation (Table 1). 
Table 1. Temperature Variation in Sahn-Yang Gotjawal's Cave Depression Throughout the Year.

\begin{tabular}{cccccccccccccc}
\hline $\begin{array}{c}\text { Temperature/Humidity } \\
\text { (By Measurement Point) }\end{array}$ & Jan. & Feb. & Mar. & Apr. & May & Jun. & Jul. & Aug. & Sep. & Oct. & Nov. & Dec. & Ave \\
\hline Ground $1.5 \mathrm{~m}\left({ }^{\circ} \mathrm{C}\right)$ & 3.5 & 4.7 & 7.0 & 11.9 & 14.3 & 16.4 & 20.9 & 21.7 & 20.0 & 17.3 & 10.7 & 7.0 & $\mathbf{1 3 . 0}$ \\
Ground $50 \mathrm{~cm}\left({ }^{\circ} \mathrm{C}\right)$ & 3.2 & 3.9 & 5.9 & 10.3 & 12.3 & 13.8 & 17.5 & 19.4 & 18.7 & 16.6 & 10.3 & 6.7 & $\mathbf{1 1 . 6}$ \\
Underground & 3.1 & 2.5 & 4.2 & 6.7 & 8.6 & 9.6 & 11.6 & 13.0 & 14.9 & 15.4 & 10.7 & 6.7 & $\mathbf{8 . 9}$ \\
$\quad 50 \mathrm{~cm}\left({ }^{\circ} \mathrm{C}\right)$ & 92.3 & 88.1 & 91.8 & 98.0 & 98.7 & 99.3 & 97.4 & 96.2 & 99.8 & 97.8 & 93.9 & 90.9 & $\mathbf{9 5 . 4}$ \\
\hline Humidity $(\%)$ & & & & & & & & & & &
\end{tabular}

\subsection{Vegetation Status}

As a result from literature review and field surveys, the vascular plants that are distributed in the Sahn-Yang Gotjawal region totaled 302 plants identified as 106 families, 220 genera, 267 species, 1 subspecies, 27 varieties and 7 breeds (Table 2).

Table 2. Vascular Plants in Sahn-Yang Gotjawal of 2019.

\begin{tabular}{cccccccc}
\hline & Family & Genus & Species & Subspecies & Variant & Breed & Subtotal \\
\hline Pteridophyta & 10 & 20 & 34 & - & 1 & - & 35 \\
Gymnosperm & 1 & 1 & 1 & - & - & - & 1 \\
Angiosperm & 95 & 199 & 232 & 1 & 26 & 7 & 266 \\
Dicotyledonous Plant & 80 & 174 & 210 & 1 & 22 & 6 & 239 \\
Monocotyledonous Plant & 7 & 25 & 22 & - & 4 & 1 & 27 \\
TOTAL & 106 & 220 & 267 & 1 & 27 & 7 & 302 \\
\hline
\end{tabular}

Along the various cave depressions in Sahn-Yang Gotjawal, the vascular plants of the region show that the Pteridophyta is distributed among 10 families, 20 genera, and 35 species. There are not many significant differences in plant distribution compared to closer Gotjawal regions. In addition, the distribution of rare plants is visible along the cave depression. It is necessary to observe plant population change continuously because plants such as the protruding spleenwort and Asian single-sorus sleepwort are limited in the Sahn-Yang Gotjawal region and are only distributed in depressions and tumulus areas. In addition, there are also other rare species that live in limited areas such as Tubiflorous Phacellanthus, common Daphniphyllum, and broad flat rock trees. These rare species only grow in unusual areas such as cave depressions. Recently, the flora in the Gotjawal are being affected by the increased amount of light and decreased shade due to the removal of dead trees with pine wilt disease.

Although Sahn-Yang Gotjawal is an excellent and unique repository of natural ecological resources, it does not have enough community development programs for residents to utilize. In addition, there is no management system, so it is necessary to establish one that not only preserves natural resources but also cooperates with the private sector. Finally, this research is conducted to propose a community development cooperation system in which nature and residents become one by expanding the eco-tourism led by programs for regional growth that contribute to the local economy.

\section{Research Method}

In this study, we sought to identify potential factors of regional community development and environmental conservation in the Sahn-Yang region and explain elements that could create synergies through media analysis and SOAR (strengths, opportunities, aspirations, results) analysis. The elements of SOAR were streamlined with social factors and ecological resources epitomized in the concept of SES. "Strengths" stands for internal and present factors. "Opportunities" covers external and present conditions. The future oriented internal factors are collected as "aspirations". "Results" provides insight based on future and external factors in Sahn-Yang Gotjawal.

The SOAR element analysis of Gotjawal in Sahn-Yang was conducted. SOAR analysis is a technique in which specific organizations derive strategic plans that focus on current strengths and 
opportunities and create a vision for future internal aspirations and the consequences they will bring. SWOT analysis has traditionally been widely used as a technique for eliciting strategic planning. SWOT and SOAR models are business management tools used as organizational frameworks to enhance business performance [35].

Many research institutes or business consulting companies have recently used both methods. SWOT analysis is an abbreviation for strengths, weaknesses, opportunities, and threats, while SOAR analysis is an abbreviation for strengths, opportunities, aspirations, and results (Figure 3). SOAR analysis has a broader range of perspectives than SWOT analysis because it can also predict future points and external elements [36]. Integration with quantitative analysis is also possible. Additionally, SOAR analysis looks positively at offering planning strategies for current perspectives of the program and future and perspectives [37]. It will help not only to get rid of the problem, but also to focus on the strengths and opportunities that can eventually change the balance in favor of the strengths [37]. The SOAR analysis improves strategic planning and implementation procedures by aiming to achieve strengths, opportunities, aspirations, and measurable results [38]. It also helps to imagine the most ideal future, making innovative strategies, programs, systems, structures, build a sustainable environment, and inspire organizational stakeholders to enhance performance and execution of strategy [38].

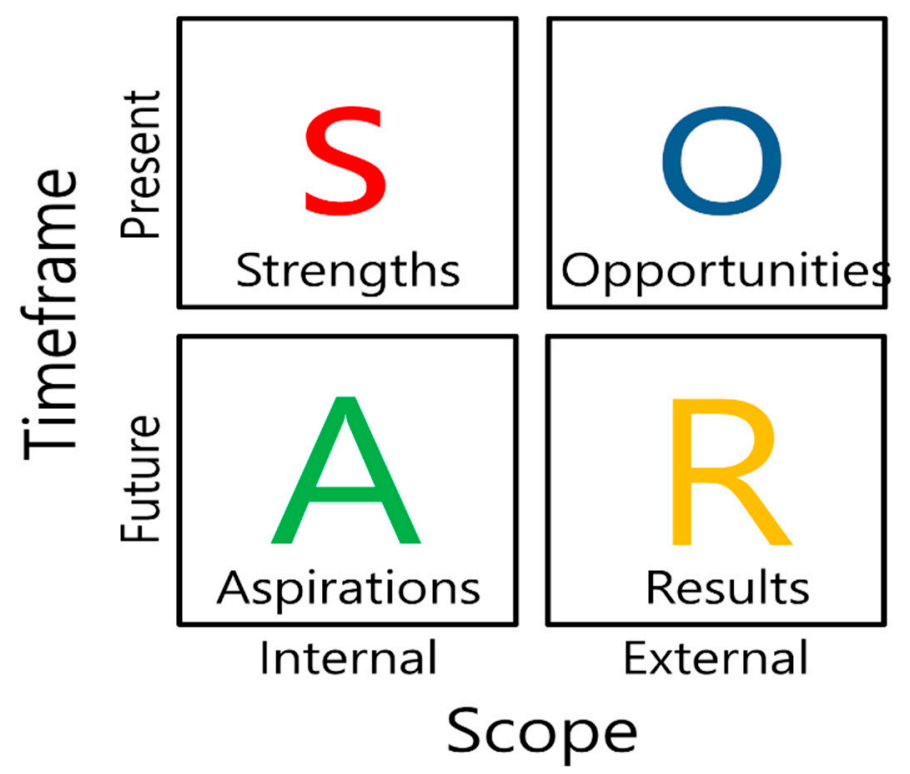

Figure 3. Strengths, Opportunities, Aspirations and Results (SOAR) Analysis Matrix.

In this study, media analysis based on newspaper articles about Gotjawal was conducted first to organize the image shown in Jeju by Gotjawal. Media analysis has many benefits for case study research projects. The researchers would be able to understand geographic selectivity and interests-focused organization in content analysis. In addition, the information obtained from complex media tools can be categorized and coded through one coherent viewpoint. The data in media holds a characteristic angle with a wide range of editorial content aimed at a broad audience. We conducted big data keyword-oriented media analysis with 5612 articles (filtered out of 8014), which were published from 1 January 2000 until 31 December 2019, by utilizing a qualitative analysis tool, Dedoose (analyzed within the platform of 2019). These 5612 articles were selected and processed in this analysis because the contents in these news reports could directly depict the factors of socio-ecological systems (SESs). Further, numerous news articles (5017) were issued in Jeju local media, including internet social media. Therefore, the media analysis was able to precisely target the local context of Jeju Gotjawal.

SOAR analysis was designed based on the results of the previous media analysis and current vegetation resources. Only the mixed method SOAR analysis method was applied. This study presents 
how SOAR analysis can demonstrate its advantages in assessing and proposing the potential of regional development.

SOAR analysis, which was conducted via surveys of 16 Gotjawal experts, is based on the contribution of the local community development programs, divided into four sections: strengths, opportunities, aspirations, and results. Each section was assigned 10 points with an "absolute evaluation" method. The priority among the four factors, which are sub-categories of each section, was relatively evaluated as giving a score of four points for first place, three points for second place, two points for third place, and one point for fourth place. For each factor, the evaluated ranking score was multiplied by the absolute evaluation score obtained through the contribution evaluation and the scores between the factors were compared having 100 points in each section. At the same time, it was possible to quantitatively determine which sections, among strengths, opportunities, aspirations, and results, to focus on and make strategies in the future. The other advantage of this SOAR analysis is that it was able to find the relative importance value of each sub-element under each section. SOAR index scores were calculated as follows.

$$
\text { Factor x's SOAR Index }=\frac{\sum_{n=1}^{P}\left\{\left[(T+1)-r_{n}\right] * E_{n}\right\}}{P}
$$

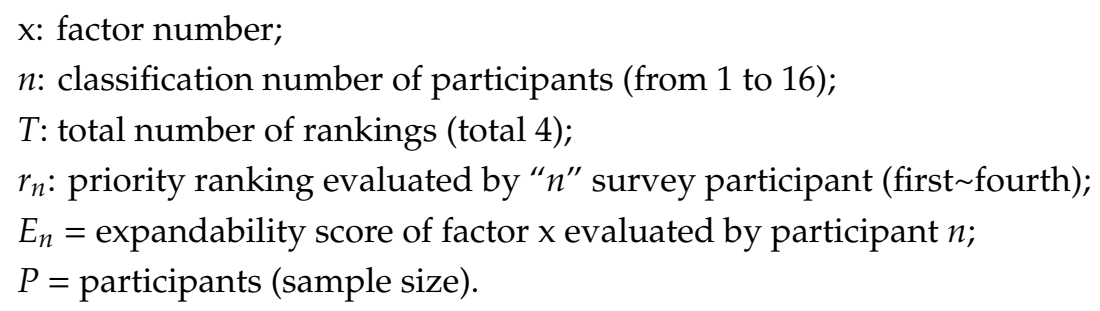

Based on the results from the designed media analysis and SOAR analysis, this research finds eco-tourism related ecological resource utilization and conservation programs that can manage and preserve Sahn-Yang Gotajwal's ecology in Jeju. In addition, it attempts to present a vision for ecological resources that can contribute to Sahn-Yang residents. In addition, the research results were intended to be used as an integrated tool that links the lives of residents in Sahn-Yang and ecological resources.

\section{Results}

\subsection{Media Analysis Results}

In this research, the media analysis frame was used to search for articles that are related to Gotjawal throughout both the Sahn-Yang area and Jeju Island as a whole. The contents were coded around keywords and evaluated based on weight and frequency. The results are as shown in the following Table 3.

To sum up the results of the media analysis, Gotjawal was a symbol of the value of "clean Jeju". In particular, the Sahn-Yang Gotjawal area was known as the representative habitat of Jeju Sahn-Yang Daphne Kiusiana Miquel. Many articles were found saying that the Sahn-Yang area has the potential to contribute to global climate element research through cooperation with leading local climate change research institutes, including the Forest Research Institute in the Republic of Korea. Thus, this shows that it is a better avenue to explore than the Kiusiana of any other region.

Even at the center of the conflict between development and conservation, the word Gotjawal was frequently searched in news articles. It may be a result reflecting the social conflict over the Gotjawal border. Another conflict is that articles related to Gotjawal, which is in private land, have been searched. It was also possible to derive that the central role of public-private cooperation and social integration is most important in terms of private forest management within the Gotjawal of Sahn-Yang. 
Table 3. Media analysis results regarding Sahn-Yang Gotjawal of 2019.

\begin{tabular}{cccc}
\hline Ranking & Keyword & Weight & $\begin{array}{c}\text { Frequency } \\
\text { (Keyword Included) }\end{array}$ \\
\hline 1 & Jeju & 24.79 & 1132 \\
\hline 2 & National Parks in Jeju & 10.2 & 112 \\
\hline 3 & Jeju white Daphne & 8 & 56 \\
\hline 4 & (Warm Temperate and Subtropical Forest Research Center) & 6 & 8 \\
\hline 5 & Private Estate & 5.87 & 46 \\
\hline 6 & Private Forest Purchase & 5.83 & 18 \\
\hline 7 & Development Activity & 5.33 & 14 \\
\hline 8 & Residents & 5.31 & 56 \\
\hline 9 & Environment Resource Total Volume System & 4.36 & 46 \\
\hline 10 & (No Net Loss Environment Policy) & 3.53 & 18 \\
\hline
\end{tabular}

\subsection{SOAR Analysis: Qualitative Analysis}

To design the survey of experts, this qualitative analysis sought to find elements such as strengths, opportunities, aspirations, and results in Sahn-Yang Gotjawal from the viewpoints of eco-tourism, based on the keyword results of the media analysis.

\subsubsection{Strengths (Present + Internal)}

The Repository of Ecological Resources Representing the Volcanic Area and Possibility of Systematic Conservation

Sahn-Yang Gotjawal serves as an ecological axis linking the western ridge of Halla Mountain to the coast. It has natural ecological resources that display the original forest of the Jeju volcanic era (both coniferous forest and broad-leaved forest). In addition, it is the habitat of endangered plants, and has regional characteristics of the Sumgol area and developed depression areas, which are particularly noticeable. Its conditions are advantageous for the growth of white Daphne and broad flat rock trees, which are exclusive species to Jeju Island. These ecological characteristics are well preserved and trails that formed naturally exist without man-made damage to the geographic features.

\section{Accessible Conditions for Visitors}

Sahn-Yang Gotjawal has the advantage of being accessible on foot. Towns around Sahn-Yang Gotjawal are also accessible because they are connected to the Gotjawal Natural Forest through Olle roads. These Olle roads are flat and they can be accessed by wheelchairs. The roads reach nearby ocean parks, so visitors can conveniently enjoy beach and forest activities in one day. The roads are also well maintained and provide quick automobile access to Aewol and Seogwipo's tourist areas. In this perspective, Sahn-Yang Gotjawal is more favorable than the more well-known Cheongsu Gotjawal or Gotajwal Provincial Park in the adjacent region.

\section{Rich Artistic and Cultural Conditions Held by Sahn-Yang Village}

Villages around Sahn-Yang Gotjawal are representative communities with historical nomadic cultural resources. The only pottery kiln built during the Joseon Dynasty was found in the Gotjawal region. Various folktales, such as the lotus pond and the dragon in Suryong-dong took place there. It is possible to establish artistic visitor programs because many artists live in nearby villages. Sahn-Yang 
Village provides an artistic experience to visitors with its art theme park $500 \mathrm{~m}$ away from the entrance of Sahn-Yang Gotjawal.

The Attraction of Sahn-Yang Village, the Center of Healing, as a Health and Longevity Village

As the forest of the broad flat rock tree (Maesa japonica) is formed, there is a marketing advantage because of a carbon sink called the fine dust reduction effect. In addition, high-sweet Sahn-Yang citrus grow near Gotjawal, which has good soil and abundant sunshine, also plays a role in the development of the local agricultural industry. Sahn-Yang Gotjawal, known as the Longevity Village, has a strength that can be marketed as the center of health and healing. It can be a powerful driver to become the center of healing, as well as having a longevity food experience program in conjunction with nearby local restaurants.

\subsubsection{Opportunities (Present + External)}

The Need for an Ecological Health Experience Program and Biological Research Using Broad Flat Rock Tree (Maesa japonica)

Residents and visitors of Jeju are becoming more interested in ecological experiences due to urban pollution and damage to natural resources. It is possible to provide health programs to residents and visitors thanks to broad flat rock trees, which mainly inhabit the Sahn-Yang Gotjawal area and reduce pollution. There is a higher expectation for the broad flat rock tree research because fine dust is emerging as a major environmental issue.

In addition, these trees can control humidity, which can greatly contribute to the study of plant walls, so there is potential for economic opportunity. In the future, it is feasible to build a database for systematic conservation of broad flat rock trees that contributes to the local industry by selling large amounts of broad flat rock tree seedlings. Since the Sahn-Yang Gotjwal region has a better distribution of broad flat rock trees than other Gotjawal regions, it would be helpful to establish a strategy of harvesting their healing function to develop and preserve Sahn-Yang Gotjawal's resources.

Demand for Flower Path Exploration: Utilization of White Daphne Resources, the Attraction of Visiting Sahn-Yang Gotjawal

In addition to the healing function of the broad flat rock tree, white Daphnes should provide the opportunity to heal. Since there is a theory that the white Daphne is indigenous to the Sahn-Yang Gotjawal area in Jeju, it should be branded as "Sahn-Yang White Daphne" in order to market the region and create future economic opportunities. As Jeju Sahn-Yang's White Daphne is different from that of Japan or China, and it has a unique scent and shape, it can be named Jeju's unique Sahn-Yang White Daphne and be used for extensive research and promotion. Since "Daphne (瑞香)" is derived from "flowers with auspicious scent from Buddha", it can be an attractive selling point for Buddhists.

Increase of Visitors to Hankyung Regional Marine Park: Research and Business Potentials that Can Be Linked to Diverse Natural Resources from Hankyung-myeon Area

Visitors who go to the Hankyung-myeon area not only visit the marine park but also other close ecological parks and forest bath areas. Thus, it is possible to form an image related to ecological resources from Hankyung-Myeon. For example, the Hankyung Ecological Exploration Map, a village tour that provides the theme of self-reflection and a walking experience that can be only felt in Sahn-Yang Gotjwal. With the global trend in banning plastics and an eco-friendly lifestyle, Hankyung's ocean, volcanic features, art, and human resources can be reinterpreted. It can also be developed as a potential key point in terms of regional industrial development. Eventually, it could be possible to install a Hankyung nature corridor belt, focused on the Sahn-Yang area, which can exhibit Jeju's beautiful scenery. 
Increased Time Spent Staying in the Sahn-Yang Area following the Expansion of Convenient Facilities

Recently, many places have attracted visitors by providing good restaurants, art facilities, and great scenery to take pictures. There is a large possibility of expanding the "potential stay period" for visitors from Aewol, Jeju cities, and the Jungmun Tourist Complex to experience forest resources in the Sahn-Yang Gotjawal area and even explore the western coast area. The west coast hidden places in Jeju such as Chagwi Island should be promoted as the spotlight of the center of Jeju's ecological environment. There is enormous potential to contribute to the revitalization of the local economy by promoting convenience and entertainment facilities that can provide visitors with opportunities for ecological exploration and healing in the Sahn-Yang area-the center of the beautiful coastline of western Jeju.

\subsubsection{Aspirations (Future + Internal)}

\section{Research on the Broad Flat Rock Tree and White Daphne}

To utilize the effectiveness of broad flat rock trees and white Daphnes, the following programs are required. First, the international distribution of broad flat rock tree and academic monitoring should be conducted. Cooperation with leading research institutes is essential, and there must be an approach utilizing professional human resources from organizations such as local universities and regional research institutes. In addition, there must be international cooperation for mass cultivation of broad flat rock tree seedlings, and through this, it is necessary to conduct a business feasibility analysis of broad flat rock trees as part of the fine dust reduction project. Moreover, by monitoring the flowering time of white Daphnes and broad flat rock trees, it will be possible to find out how climate change in Jeju and other western Pacific regions is progressing and how it affects the natural ecosystem.

\section{Serve as the Center of the Tree Planting Movement for Green Jeju (Leading Jeju Campaign)}

The following programs are needed to create a haven for not only broad flat rock trees, but also Korean Dendropanax morbiferus inhabiting Jeju's volcanic forest area. First, through the tree planting campaign, villages around the Sahn-Yang Gotjawal area should be expanded to serve as the center of the entire Jeju society. It is worth considering establishing online promotion so that the tree-planting movement can be recognized as one of the representative experience programs in Jeju. Furthermore, if the Jeju Sahn-Yang tree planting contest, which can be considered as a festival-type Arbor day event, is held as a representative campaign of "Green" Jeju island, it can be turned into a local attraction. This can become a new local culture by introducing the health benefits of broad flat rock trees and tree planting campaigns on in-flight magazines with Korean domestic airlines such as Jeju Air, Korean Air, and Asiana Airlines so that visitors who travel to Sahn-Yang, Jeju, or Korea can learn about Jeju's broad flat rock tree benefits.

\section{Formation of Sahn-Yang Ceramics Art Village}

To form the Sahn-Yang Ceramics Art Village, the following programs are necessary. First, hands-on programs to design and make pots under the forest of broad flat rock trees, which are sold to continue the culture of pottery production of the Joseon Dynasty. Second, domestic, and foreign ceramic expert exchange programs should be provided to help it become a mecca of the traditional ceramic pottery field. Third, developing the art village town can be initiated from proposing and promoting strategies and specific projects to brand Sahn-Yang ceramics. Lastly, research on ceramic products that can reduce the use of plastic is needed and will lead to a sustainable art culture that creates eco-friendly artworks.

\section{Bionic Research for Technological Innovation}

It is possible to conduct bionic research using climate change resources in the Sumgoal (underground water path) area. Researchers are able to monitor humidity and temperature changes in 
the Gotjawal area, which will assist climate change research, and it will help create an environment for experiments on plants and animals. In addition, the cooperation with international research institutes can lead to the reputation of an eco-friendly eco-tourism site in Sahn-Yang Gotjawal. Once Sahn-Yang Gotjawal gets the spotlight as a center of climate change research and an eco-friendly tourism site, it can be featured and advertised as a health recovery program in internationally famous journals like U.S. News and Healthy Aging.

\subsubsection{Results (Future + External)}

\section{Attracting Student Exploration Teams Using Access to English Education City Schools}

To expand the global level of ecological experience education, which is tailored to the educational curriculum of international schools using Gotjawal resources, the following programs are needed. First, a program that can help students understand the vegetation structure of Gotjawal while observing the vegetation resources. Through this program, students will gain a sense of balance in development and preservation. Ultimately, it will function as a natural learning center for making leaders in a globalized society and it will enhance international competitiveness for ecological education in Jeju. Furthermore, it will offer a place for children to imagine an eco-friendly future by experiencing the importance of nature via a playground against a backdrop of broad flat rock trees and white Daphne forest.

\section{Revitalize the Local Economy by Increasing the Number of Visitors in the Sahn-Yang Area}

The following programs can be managed to increase the number of visitors in the Sahn-Yang area. First, the high-quality exploration program where healing and art meet can create a desire while promoting artistry and health. Connecting through Gotjawal Art Village, this harmonious hybrid program with nature and art will be offered to visitors. This will motivate artists and promote artistic innovation. At the same time, visitors can come to experience a nature therapy forest bath to heal as they stay in the Sahn-Yang village helping to revitalize the local economy. As a result, environmental ecological jobs will be created for the conservation and sustainable use of Gotjawal's ecological resources and the income sources of residents can also broaden.

Sahn-Yang Gotjawal, Established as a Symbol of Longevity

To succeed with "the symbol of longevity Sahn-Yang Gotjwal", the following projects are needed. Jeju's eco-friendly Sahn-Yang Gotjawal's branding should be completed. In addition, it is necessary to develop a visitor stay program that seeks to restore health from the fine dust problem. A Sahn-Yang experience program for Jeju local residents should be promoted and an international evaluation of the medical treatment efficacy of broad flat rock trees could also be conducted to support the program.

\section{Gotjawal Road, as the World Healthy Flower Road}

Gotjwal Road needs the following programs to be a "world healthy flower road". Many festivals can be started based on traditional folktales. For example, the story of Buddha related to the white Daphnes in Sahn-Yang Gotjawal can be a nice attraction for tourists. During the winter season, which is the off season in Jeju Island, white Daphne thrive so a white Daphne festival can boost the local economy. This white Daphne festival should have specific strategies and business programs to attract visitors who seek mental healing and romance during the winter season. In addition, Gotjwal Road can achieve ecological tourism as a healthy natural trail that families and couples can enjoy together. Lastly, a new refined vision is needed to regenerate itself as a place to relieve stress for busy city visitors and as a romantic flower road that can be enjoyed even in the winter season. 


\subsection{SOAR Analysis: Quantitative Analysis}

Based on the result of the SOAR quantitative analysis, it concluded that projects that have higher contributions for business should be promoted (strengths section's SOAR Index $=86.5 / 100$ ), and from each factor of the section, it was first agreed to systematically conserve ecological resources of Sahn-Yang Gotjwal such as broad flat rock trees and white Daphnes (SOAR Index = 35.28). Next, a study on the broad flat rock trees and white Daphnes (SOAR Index $=29.12$ ) should be planned to be utilized as an ecological health experience program and a local ecological education site. In other words, to sustain and utilize Sahn-Yang Gotjwal resources is to promote the subjects of health and education and pioneer the programs. The participants of the survey had a higher expectation of potentials of Sahn-Yang Gotjwal's resources to represent not only the Sahn-Yang area but also Jeju's entire area as a center of health, healing, and environment. In addition, they suggested that visitors should be given a satisfying experience through the creation of the global ecological education site and Gotjwal Road by utilizing the resources from Sahn-Yang Gotjwal (SOAR Index $=28.43$ ).

In the SOAR study, 16 experts were given a priority-oriented SOAR analysis survey, and the results were as follows:

The sum of the points of Tables 4-7, and the summary of qualitative analysis through media analysis and SOAR analysis, are as follows: First, it was concluded that a strategy is needed to provide exploration programs by utilizing eco-forest classrooms, while systematically managing and conserving ecological resources of the Sahn-Yang Gotjawal area. To realize this, it is necessary to preserve it as a habitat for endangered wild animals and plants as it is the center of the ecological axis linking the western ridge and coast of Hallasan Mountain. Sahn-Yang Gotjawal is well preserved compared to other regions, and it has the potential to expand into the scientific research fields around climate change and cave depressive areas because of the unique volcanic geological zone. It has optimal conditions for Sahn-Yang broad flat rock trees and white Daphnes and provides a unique healing tour opportunity for visitors. In addition, white Daphnes and broad flat rock trees can be utilized in medical experiments which can contribute to the global community.

Table 4. Strengths: A Healing Space Should be Created with the Sahn-Yang Gotjawal's Ecological Resources.

\begin{tabular}{|c|c|c|}
\hline & Section 1: Strengths (Present + Internal) & SOAR Index \\
\hline Factor 1 & $\begin{array}{l}\text { The repository of ecological resources representing the volcanic area and } \\
\text { possibility of systematic conservation }\end{array}$ & 35.28 \\
\hline Factor 2 & Accessible conditions for visitors & 18.66 \\
\hline Factor 3 & Rich artistic and cultural heritage held by Sahn-Yang Village & 20.15 \\
\hline \multirow[t]{2}{*}{ Factor 4} & $\begin{array}{l}\text { The attraction of Sahn-Yang Village, the center of healing, as Healthy } \\
\text { Longevity Village }\end{array}$ & 12.36 \\
\hline & 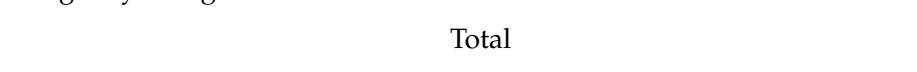 & $\begin{array}{c}86.46 \\
\text { (out of } 100)\end{array}$ \\
\hline
\end{tabular}

Table 5. Opportunities: in order to turn the current external situation into an opportunity, it is necessary to focus on the center of ecological tourism and conservation of natural resources in the Hankyung-myeon area by making a broad flat rock tree and white Daphne road.

\begin{tabular}{|c|c|c|}
\hline & Section 2: Opportunities (Present + External) & SOAR Index \\
\hline Factor 5 & $\begin{array}{l}\text { Need for ecological health experience program and biological research using } \\
\text { broad flat rock trees }\end{array}$ & 17.82 \\
\hline Factor 6 & $\begin{array}{l}\text { Demand for flower path exploration: utilization of White Daphne resources, } \\
\text { the attraction of visiting Sahn-Yang Gotjawal }\end{array}$ & 25.64 \\
\hline Factor 7 & $\begin{array}{l}\text { Increase of visitors to Hankyung Regional Marine Park: research and } \\
\text { business potentials that can be linked to diverse natural resources from the } \\
\text { Hankyung-Myeon area }\end{array}$ & 20.56 \\
\hline \multirow[t]{2}{*}{ Factor 8} & $\begin{array}{l}\text { Increased time spent staying in the Sahn-Yang area due to the expansion of } \\
\text { convenience facilities }\end{array}$ & 15.21 \\
\hline & (1) & $\begin{array}{c}79.25 \\
\text { (out of } 100)\end{array}$ \\
\hline
\end{tabular}


Table 6. Aspirations: studying broad flat rock trees and white Daphnes, the ecological resources of Sahn-Yang Gotjawal, is the future of Sahn-Yang.

\begin{tabular}{|c|c|c|}
\hline & Section 3: Aspirations (Future + Internal) & SOAR Index \\
\hline Factor 9 & Research on the broad flat rock tree and White Daphne & 29.19 \\
\hline Factor 10 & $\begin{array}{l}\text { Serve as the center of the tree planting movement for Green Jeju (Leading } \\
\text { Jeju Campaign) }\end{array}$ & 21.79 \\
\hline Factor 11 & Formation of Sahn-Yang Ceramics Art Village & 14 \\
\hline \multirow[t]{2}{*}{ Factor 12} & Bionic research for technological innovation & 12.77 \\
\hline & Total & $\begin{array}{c}77.76 \\
\text { (out of } 100)\end{array}$ \\
\hline
\end{tabular}

Table 7. Results: ultimately, creating the Gotjawal Road through a focus on the attractiveness of Sahn-Yang Gotjawal ecological resources such as broad flat rock trees and white dahpnes plus utilizing ecological health experience programs will lead to a center for environmental longevity and ecological education as well as a hub for environmental capital.

\begin{tabular}{|c|c|c|}
\hline & Section 4: Results (Future + External) & SOAR Index \\
\hline Factor 13 & $\begin{array}{l}\text { Attracting student exploration teams using access to English education } \\
\text { city schools }\end{array}$ & 11.27 \\
\hline Factor 14 & $\begin{array}{l}\text { Revitalize local economy by the increased number of visitors in } \\
\text { Sahn-Yang area }\end{array}$ & 23.03 \\
\hline Factor 15 & Sahn-Yang Gotjawal, established as a symbol of longevity & 17.96 \\
\hline \multirow[t]{2}{*}{ Factor 16} & Gotjawal Road, the world healthy flower road & 28.43 \\
\hline & , & $\begin{array}{c}80.70 \\
\text { (out of } 100)\end{array}$ \\
\hline
\end{tabular}

Second, various methods were applied to improve economy of the Sahn-Yang area by utilizing natural ecological resources in Sahn-Yang Gotjwal in a sustainable way while conserving the resources in a future oriented way. Through SOAR analysis, various considerations of potential situations, and potential use of the Sahn-Yang Gotjawal resources were imputed and analyzed with quantitative and qualitative techniques. In addition, through media analysis, the benefits, and opportunities that the Gotjawal resources could give to citizens were identified and the results were combined with SOAR analysis to present an integrated vision.

Third, programs for visitors and residents were created so they could enjoy and preserve the Sahn-Yang Gotjawal resources. In addition, Sahn-Yang Gotjawal's 2030 vision was suggested and created as the groundwork for the second research task. The 2030 vision of Sahn-Yang Gotjwal is a vision of co-prosperity and integration with residents, which aims to take the future in a sustainable direction, focusing on three factors: 'recovery', 'balance', and 'inclusion'.

\section{Discussion}

People in Jeju Island wish to establish an ecological conservation management system for the Sahn-Yang Gotjawal ecological resources deserving of Jeju's international brand. However, the policy measures are insufficient in terms of the development of community-led programs that can coexist with residents. Specifically, it is time to appreciate 'purity' and 'coexistence', which are the core values of Jeju's Future Vision [39], and time to propose a plan to implement the Gotjawal ecological program systematically to preserve, manage, and utilize the ecological assets of Sahn-Yang Gotjawal.

The number of visitors is steadily increasing after Gotjawal was introduced through the media. The management authority and systematic utilization programs need to be improved to match the increase in visitors. Gotjawal's current situation and problems lie in defining what most residents and administrative authorities want to utilize from Gotjawal resources. Developing an ecological program by reorganizing the eco trail of the forgotten Gotjawal, finding undiscovered materials, and creating community development programs feasible with residents' income are the present issues. There were 
advantages from the movement to preserve geological features and the ecosystem of wild animals and plants. However, and unfortunately, the negative effect of only seeking development opportunities without regard to the environment was realized as well. Unrefined development activities and pressures taking place outside and around Gotjawal have also raised concerns about environmental changes in Sahn-Yang Gotjawal.

Sahn-Yang Gotjawal, therefore, needs to prepare comprehensive tourism plans and strategies to implement sustainable development. According to Khavarian-Garmsir [38], tourism activities should center on a type of resources management in which all economic, social, and aesthetic requirements are considered alongside cultural integrity, essential ecological processes, and biological diversity. It is necessary to bring up all aspects of tourism for comprehensive planning [37]. Strategic planning helps to set goals of tourism development while deciding and implementing measures constructively to assist the decision-making process of development [37]. These strategies for sustainable development must be made and implemented in a realistic, effective, and lasting way [38].

Jeju Sahn-Yang Gotjawal also needs an attitude and approach for preserving and utilizing resources based on the perception that it is a heritage of the past, present, and future in terms of diversity of Gotjawal ecology. In addition, we should regard the value of the village as a public good. In order to make wise use of Sahn-Yang Gotjawal, such as conservation, sustainability, and accessibility (guided by SESs), it is necessary to create a hub center, which can develop management programs such as preservation research and monitoring, as well as establishing sustainable usage of facilities, such as visitors' gathering spaces, exploration activities, and convenient facilities. To provide high-quality exploration services to visitors as the hub center, it is necessary to promote awareness of Gotjawal and a service space to provide comprehensive ecological information and practical reasons to visit Sahn-Yang Gotjawal

\subsection{Eco-Tourism Direction for Residents Using Sahn-Yang Gotjawal Resources: Robustness}

In this study, a future-oriented eco-tourism direction was created with the residents through understanding and utilizing of resources from Sahn-Yang Gotjawal. As a result, Sahn-Yang Gotjawal is more competitive than the regions that are currently operating visitor programs using volcanic resources.

For Sahn-Yang Gotjawal's competitiveness, a concrete feasibility assessment formed by the proposed projects and sustainable spatial expansion plans should be established in the future. Specifically, Jeju's clean zone, which is promoted as the world's environmental hub, needs particular attention given to Sahn-Yang Gotjawal, and the Sahn-Yang as those are the center of the environment capital. Environmental solutions such as establishing Jeju National Park (now Jeju holds only Mt. Halla National Park) and the environmental resource quota system (no net loss in ecological resources) can also serve as a systematic monitoring site provided by Sahn-Yang Gotjawal (contributing to DB).

To achieve eco-tourism using the Sahn-Yang Gotjawal resources, it is necessary to create a Gotjawal Road with the Jeju white Daphne as an amenity provided for visitors and residents. To do this, the landscape and program provided by Sahn-Yang Gotjawal should be upgraded. It is also necessary to establish a monitoring database that can provide important data for climate change research as well as forming a self-governing alliance for conserving and managing Sahn-Yang Gotjawal by cooperating with Jeju Residents' Environmental Self-Governing Alliance and NGOs.

Above all, the title of Sahn-Yang Gotjawal, which is linked to the internationally renowned the United Nations Educational, Scientific and Cultural Organization (UNESCO) triple crown (world natural heritage site, world natural heritage site, geopark) is important. Therefore, measures should be taken to strengthen consultation and research capabilities for word of mouth advertising.

\subsection{Restoration of Cleanliness Using the Sahn-Yang Gotjawal Resources: Resilient Restoration}

Transparency regarding the nature of the ecological resources of Sahn-Yang Gotjawal is vital to establish a recovery strategy to create future-oriented values. The installation of the Gotjawal Road, which consists of the main resources of Sahn-Yang Gotjawal such as broad flat rock trees and white 
Daphnes, should provide a foundation for the conservation and maintenance of ecological resources as well as restoring damage to volcanic geological areas in the future.

To expand the monitoring system for ecological recovery, a reasonable policy-making debate is needed. The capacity to manage and maintain the diversity of vegetation and animal species in the Gotjawal region must be met. While improving the damaged natural ecological system, it is necessary to maximize the expected profits when the total environmental resource system is introduced. This can be done by establishing a sustainable land use system for environmental conservation. An ecological sampling checklist related to the broad flat rock tree and white Daphne is needed and a system for international certification and evaluation should be established as well.

\subsection{Balance of Coexistence and Cleanliness, Development and Conservation, Sahn-Yang Gotjawal: Conformable and Sustainable Locality: Sustainability}

To establish a sustainable utilization plan so that the Sahn-Yang Gotjawal area can serve as a natural park and a base for preserving the ecosystem, it is necessary to conduct a survey of land use and a systematic evaluation record of the facilities that can be set up as a park and as a research facility. From a global perspective, extensive research is needed on Gotjawal related to the study of climate change response, the use and reprocessing of groundwater and water resources, and conservation.

In addition, a balance between social factors and ecological resources should be established based on research results on the resilience and development of local culture that enhances the value of vegetation while ensuring the safety and happiness of visitors (Broad Flat Rock Tree Road, which represents "healing" and Sahn-Yang Flower Road, which has white Daphnes which stand for "peaceful love"). To build a balanced vision, ecological services from the resources of Gotjawal and ecosystem equilibrium evaluation research, should be conducted. In addition to fostering community development projects for preserving ecological resources, reputation and image management are also needed to establish Sahn-Yang Gotjawal as a global environmental brand.

\subsection{Cooperative and Deliberative Regional Management System: Symbol of Engagement, Sahn-Yang Gotjawal: Governance}

To analyze social factors in the Sahn-Yang area and establish a community identity that can be promoted by the Sahn-Yang Gotjawal resources, the capacity of the Gotjawal resource management must be strengthened and promote a campaign to preserve Gotjawal with cooperation between residents and visitors. Through healthy conflict resolution and ecological resource management, Gotjawal can be connected with other Gotjawal parks but also act as a hub in Jeju Gotjawal management network.

\subsection{The Center of the Environmental Capital, Sahn-Yang Gotjawal: The Future of Jeju: Adaptive Management}

The longevity of Gotjawal resources through proper monthly visitor simulation surveys is needed to promote the field of global-oriented vegetation resource education. In addition, spatial analysis modeling and climate change data can be applied to cope with future risks of Sahn-Yang Gotjawal. Specifically, it is necessary to introduce the World Natural Heritage Headquarter's global strategy elements to the Sah-Yang Gotjawal exploration and management program and establish Sahn-Yang as an international ecological hub. It is meaningful to study the existing infrastructure and future demand in the Sahn-Yang region in order to establish research that matches ecological preservation and utilization strategies, and to establish an adaptive Gotjawal management model that simultaneously combines scientific progress and social integration.

\section{Conclusions}

This study covers a systematic and quantitative assessment of the function, design, and purpose of interpreting eco-tourism using Jeju San-Yang Gotjawal natural resources. Our SOAR analysis research (SOAR score: strength 86.46 ) with professionals found that a healing space for visitors should 
be created with the Sahn-Yang Gotjawal's ecological resources for successful regional eco-tourism program development.

The future academic task of this study is to make suggestions that provide research direction for the governance of community development by utilizing Sahn-Yang Gotjawal eco-tourism resources. First, an integrated policy development model is necessary. This is related to the efficiency, rationality, and feasibility of database management for the use of environmental ecological resources in Sahn-Yang Gotjawal. It is vital to consider the governance design to improve the functionality and efficiency of governance that can systematically manage Gotjawal ecological resources and evaluate relevance. In addition, a strategy is needed to match the demands of visitors and tourists with the sustainable charm of Sahn-Yang Gotjawal.

Second, as a representative of the Jeju volcanic geological zone, it is necessary to implement a policy that emphasizes conservation as a geological and biodiversity maintenance habitat in Sahn-Yang Gotjawal. Cooperation is needed with specialized institutions to increase the efficiency of Gotjawal management governance. In addition, condition analysis is needed for strengthening the management system of vegetation resources (such as Broad Flat Rock trees and white Daphnes) and establishing a monitoring system that considers the landscape and ecological characteristics of Gotjawal. This benefits education for academic specialization, strengthens autonomy, and spreads the voluntary grassroots practice campaign.

A feasibility analysis of the eco-tourism project for revitalization of urban regeneration projects and rural centers in Sahn-Yang should be periodically conducted. A Gotjawal community development council needs to embrace the local residents and experts who research Gotjawal. Further, and finally, no one can deny the importance of the public and private sector's diversified collaborative support for Sahn-Yang Gotjawal.

Author Contributions: C.-Y.H. and R.Y. provided direction to the research work and participated in the research. J.-D.H. did the literature review and collected relevant data and C.-Y.H. wrote the manuscript. In addition, M.-S.J. searched and collected data through the field survey; he searched and collected literature and evidence. C.-Y.H. and R.Y. revised the paper. M.-S.J. and J.-D.H. collected and analyzed the literature this paper needs. All authors have read and agreed to the published version of the manuscript.

Funding: This research received no external funding.

Acknowledgments: We appreciate the productive suggestion from editors and anonymous reviewers and would like to give our thanks to them.

Conflicts of Interest: The authors declare no conflict of interest.

\section{References}

1. Stone, M.; Wall, G. Ecotourism and community development: Case studies from Hainan, China. Environ. Manag. 2004, 33, 12-24. [CrossRef] [PubMed]

2. Cater, E. Ecotourism: A sustainable option? In Ecotourism in the Third World: Problems and Prospects for Sustainability; Cater, E., Lowman, G., Eds.; John Wiley \&Sons: Chichester, UK, 1994; pp. 69-86.

3. Ceballos-Lascurain, H. Ecotourism as a worldwide phenomenon. In Ecotourism: A Guide for Planners and Managers; Lindberg, K., Hawkins, D.E., Eds.; Ecotourism Society: North Bennington, VT, USA, 1993; pp. 12-14.

4. Western, D. Ecosystem Conservation and Rural Development: The Amboseli Case Study; Liz Claiborne Art Ortenberg Foundation: New York, NY, USA, 1993; p. 48.

5. Whelan, T. Ecotourism and its role in sustainable development. In Nature Tourism: Managing for the Environment; Island Press: Washington, DC, USA, 1991; pp. 3-22.

6. Boo, E. Ecotourism: The Potentials and Pitfalls, Country Case Studies; World Wildlife Fund: Washington, DC, USA, 1990; Volume 2.

7. Stronza, A.; Hunt, C.; Fitzgerald, L. Ecotourism for Conservation. Annu. Rev. Environ. Resour. 2019, 44, 229-253. [CrossRef]

8. Hunt, C.A.; Harbor, L.C. Pro-environmental tourism: Lessons from adventure, wellness and eco-tourism (AWE) in Costa Rica. J. Outdoor Recreat. Tour. 2019, 28, 1-8. [CrossRef] 
9. Hiwasaki, L. Community-Based Tourism: A Pathway to Sustainability for Japan's Protected Areas. Soc. Nat. Resour. 2006, 19, 675-692. [CrossRef]

10. Choi, H.C.; Sirakaya, E. Sustainability indicators for managing community tourism. Tour. Manag. 2006, 27, 1274-1289. [CrossRef]

11. Britton, S. Tourism, Capital, and Place: Towards a Critical Geography of Tourism. Environ. Plan. D Soc. Space 1991, 9, 451-478. [CrossRef]

12. Picard, D.; Robinson, M. Remaking worlds: Festivals, tourism and change. In Festivals, Tourism and Social Change: Remaking Worlds; Robinson, M., Phipps, A., Eds.; Channel View Publications: Bristol, UK, 2006; pp. 1-31.

13. Pomering, A.; Noble, G.; Johnson, L.W. Conceptualizing a contemporary marketing mix for sustainable tourism. J. Sustain. Tour. 2011, 19, 953-969. [CrossRef]

14. González, J.A.; Montes, C.; Rodríguez, J.; Tapia, W. Rethinking the Galapagos Islands as a complex social-ecological system: Implications for conservation and management. Ecol. Soc. 2008, 13. [CrossRef]

15. Operationalizing the Social-Ecological Systems Framework to Assess Sustainability. Available online: https://www.pnas.org/content/112/19/5979 (accessed on 1 October 2020).

16. Ostrom, E. A general framework for analyzing sustainability of social-ecological systems. Science 2009, 325, 419-422. [CrossRef]

17. Apostolopoulou, E.; Drakou, E.G.; Santoro, F.; Pantis, J.D. Investigating the barriers to adopting a 'human-in-nature' view in Greek biodiversity conservation. Int. J. Sustain. Dev. World Ecol. 2012, 19, 515-525. [CrossRef]

18. Anderies, J.M.; Janssen, M.A.; Ostrom, E. A framework to analyze the robustness of social-ecological systems from an institutional perspective. Ecol. Soc. 2004, 9. [CrossRef]

19. Blaikie, P.; Brown, K.; Stocking, M.; Tang, L.; Dixon, P.; Sillitoe, P. Knowledge in action: Local knowledge as a development resource and barriers to its incorporation in natural resource research and development. Agric. Syst. 1997, 55, 217-237. [CrossRef]

20. Fleischman, F.D.; Boenning, K.; Garcia-Lopez, G.A.; Mincey, S.; Schmitt-Harsh, M.; Daedlow, K.; Ostrom, E. Disturbance, response, and persistence in self-organized forested communities: Analysis of robustness and resilience in five communities in southern Indiana. Ecol. Soc. 2010, 15. [CrossRef]

21. Nassl, M.; Löffler, J. Ecosystem services in coupled social-ecological systems: Closing the cycle of service provision and societal feedback. Ambio 2015, 44, 737-749. [PubMed]

22. Gunderson, L.H.; Pritchard, L. Resilience and the Behavior of Large-Scale Systems; Island Press: Washington, DC, USA, 2012; Volume 60.

23. Berkes, F.; Folke, C.; Colding, J. Ecological practices and social mechanisms for building resilience and sustainability. In Linking Social and Ecological Systems: Management Practices and Social Mechanisms for Building Resilience; Colding, J., Ed.; Cambridge University Press: Cambridge, UK; New York, NY, USA, 1998; pp. 414-436.

24. Chaffin, B.C.; Gosnell, H.; Cosens, B.A. A decade of adaptive governance scholarship: Synthesis and future directions. Ecol. Soc. 2014, 19, 56. [CrossRef]

25. Leach, M. Re-framing Resilience: Trans-disciplinarity, Reflexivity and Progressive Sustainability-A Symposium Report, STEPS Working Paper 13; STEPS Centre: Brighton, UK, 2008.

26. Zia, A.; Hirsch, P.; Songorwa, A.; Mutekanga, D.R.; O'Connor, S.; McShane, T.; Norton, B. Cross-scale value trade-offs in managing social-ecological systems: The politics of scale in Ruaha National Park, Tanzania. Ecol. Soc. 2011, 16. [CrossRef]

27. Simpson, M.C. Community benefit tourism initiatives-A conceptual oxymoron. Tour. Manag. 2008, 29, 1-18. [CrossRef]

28. Pavlova, M. Green skills as the agenda for the competence movement in vocational and professional education. In Competence-Based Vocational and Professional Education; Mulder, M., Ed.; Springer International Publishing: New York, NY, USA, 2017; pp. 931-951.

29. Hviding, E.; Bayliss-Smith, T. Islands of Rainforest: Agroforestry, Logging and Eco-Tourism in Solomon Islands; Routledge: London, UK, 2018.

30. Gahm, S. Gotjawal Conservation and Management Plan; Ministry of Environment: New Delhi, India, 2012.

31. Ahn, W.; Choi, H. The major causes of Gotjawal formation in Jeju Island. J. Geol. Soc. Korea 2015, 51, 1-19. [CrossRef] 
32. Song, S. Distributions and Lithology of the Aa Rubble Flows on Cheju Island, Korea. Ph.D. Thesis, Pusan National University, Pusan, Korea, 2000.

33. Jeon, Y.; Ahn, W.; Ryu, C.; Kang, S.; Song, S. A review of geological characteristics of Gotjawal terrain in Jeju Island: Preliminary Study. J. Geol. Soc. Korea 2012, 48, 425-434.

34. Lee, J.; Kim, J.; Park, J.; Hong, S.; Lim, J.; Choi, H. Age of volcanic activity from Quaternary deposits in Sangchang-ri, Jeju island. J. Geol. Soc. Korea 2014, 50, 697-706. [CrossRef]

35. Gheibi, M.; Karrabi, M.; Mohammadi, A.; Dadvar, A. Controlling air pollution in a city: A perspective from SOAR-PESTLE analysis. Integr. Environ. Assess. Manag. 2018, 14, 480-488. [CrossRef] [PubMed]

36. SOARing from SWOT Four Lessons in Strategic Planning Done Right. Available online: https://spartinaconsulting. com/wp-content/uploads/2013/09/SOAR-from-SWOT_Spartina.pdf. (accessed on 1 October 2020).

37. Rashidi, M.; Hamzepour, R.; Ismaelzadeh, S.; Fateh, K. Regional Planning Tourism Strategies Based on the SOAR Approach (Case Study:Marivan City). Asian J. Water Environ. Pollut. 2018, 15, 13-21. [CrossRef]

38. Khavarian-Garmsir, A.R.; Zare, S.M. SOAR Framework as a New Model for the Strategic Planning of Sustainable Tourism. Tour. Plan. Dev. 2015, 12, 321-332. [CrossRef]

39. Kim, D.; Lee, K.; Jung, K.; Cho, J. Jeju's Future Vision Plan.: Jeju Special Self-Governing Province; Korea Research Institute for Human Settlements: Sejong, Korea, 2016.

(C) 2020 by the authors. Licensee MDPI, Basel, Switzerland. This article is an open access article distributed under the terms and conditions of the Creative Commons Attribution (CC BY) license (http://creativecommons.org/licenses/by/4.0/). 